Proceedings

\title{
Natural Aspirin-Like Compounds from White Willow (Salix Alba) Bark Extract Prevent Structural Changes of Human Hemoglobin during in Vitro Non-Enzymatic Glycation and Fructation, Preserving Its Peroxidase and Esterase Activity ${ }^{\dagger}$
}

\author{
Ionela Cristina Nica *, Maria Mernea, Gheorghe Stoian and Anca Dinischiotu \\ Department of Biochemistry and Molecular Biology, Faculty of Biology, University of Bucharest, 91-95 \\ Splaiul Independentei, 050095 Bucharest, Romania; maria.mernea@bio.unibuc.ro (M.M.); \\ gheorghe.stoian@bio.unibuc.ro (G.S.); anca.dinischiotu@bio.unibuc.ro (A.D.) \\ * Correspondence: cristinai.nica@gmail.com; Tel.: +40-213-181-575 \\ + Presented at the 1st International e-Conference on Antioxidants in Health and Disease, 1-15 December \\ 2020; Available online: https://cahd2020.sciforum.net/.
}

Published: 30 November 2020

\begin{abstract}
Proteins undergo continuous changes under the action of various intrinsic and extrinsic factors, leading to alteration of several intracellular metabolic pathways and the development of various clinical disorders. Non-enzymatic glycosylation is one of the main factors responsible for the progression of diabetic complications and the aging process. Although there are currently many effective therapies in the prevention and treatment of these diseases, in the last decade there has been an increasing trend of replacing synthetic drugs by natural compounds, in order to reduce the side effects that may occur, and also the production costs. It is well known that aspirin (acetylsalicylic acid) inhibits the glycation process of serum proteins by acetylating $\mathrm{N}$-terminal amino groups and lysine residues in their structure. Therefore, the main purpose of our research was to analyze the non-enzymatic glycation and fructation process of hemoglobin through spectrometric and electrophoretic techniques, in order to reveal how this process could influence the three-dimensional structure and biological function of the protein, and the effect of some natural aspirin-like compounds on the peroxidase and esterase activity of hemoglobin during fructose and glucose binding. In this way, a preliminary phytochemical characterization of a bark extract of white willow (Salix alba) was performed in order to evaluate the content of total phenolics, flavonoids, and salicylic derivatives, as well as the antioxidant activity. Then, human erythrocytes isolated from whole peripheral blood were incubated with different concentrations of fructose/glucose (10, 50, 100 $\mathrm{mM}$ ) and S. alba extract for 5, 7, 10 and 14 days. The results obtained from the THz spectra confirmed that fructose was more reactive than glucose, so the glycation process took place more slowly than fructation. Also, the presence of $S$. alba extract showed an antiglycosylating effect, but not a total inhibition of the glycation process. In addition, enzymatic determinations proved that willow bark extract restored the peroxidase and esterase activities to the control levels. Our data indicated that salicylic compounds can be successfully used as substitutes for aspirin, one of the main synthetic compounds with anti-inflammatory and anti-glycosylating role. Salicin, salicylic acid and other salicylic compounds possess strong antioxidant properties, which give them the ability to participate in the glycosylation process to block the formation of advanced glycation end products (AGEs).
\end{abstract}




\section{Introduction}

Protein glycation is a complex cascade of condensation, rearrangement, fragmentation, and oxidative changes; non-enzymatic glycation is a natural phenomenon, which consists in the attachment of free carbohydrate molecules to certain amino acid residues in the structure of proteins, leading to the formation of products with major alterations in secondary and tertiary structure, which also affects their functional properties. These ligation products are called AGEs [1].

Synthetic inhibitors of AGEs formation tested clinically so far have shown low efficacy, reduced pharmacokinetic properties and generated a number of side effects incompatible with patient safety, so in recent decades there has been an increasing emphasis on natural products based on plant extracts with antiglycosylating effect and safe for human consumption. In addition to their hypoglycemic properties, they also have hypolipidemic and antioxidant potential [2].

For example, salicylic acid and its derivatives possess not only anti-inflammatory properties conferred by inhibition of cyclooxygenase activity, but also an antioxidant potential that complements and potentiates the therapeutic effects of these compounds. Therefore, salicylates are well known as hydroxyl radical scavengers, their mechanism of action being based on the chelation of transition metals, regulation of nitric oxide levels, increased lipoxin synthesis with an important role in maintaining a balance of oxidative stress, and inhibition of protein kinases NF-kB and AP-1. Moreover, these compounds protect against oxidation of low-density lipoproteins (LDL) and have an inhibitory effect on the expression of lectin-like receptors for oxidized LDL on the surface of endothelial cells [3].

Starting from this similarity between acetyl salicylic acid and salicylic compounds in terms of their anti-inflammatory, analgesic and antipyretic effects, the present study aims to highlight the antiglycosylating properties of salicylic acid and its derivatives, being known that aspirin intervenes in the glycation process of proteins, preventing the formation of the final products of advanced glycation responsible for the progression of certain complications in various pathological processes (diabetes, aging, etc.).

\section{Experiments}

Willow bark (Salicis cortex) was harvested from young branches of 2 to 3 years old, then crushed, dried in a well ventilated area, and ground. The resulting powder was extracted using a solvent $(0.9 \%$ $\mathrm{NaCl})$ to sample ratio of 5:1 (v/w). After an hour of ultrasound and $10 \mathrm{~min}$ of centrifugation at 5000 rpm and $4{ }^{\circ} \mathrm{C}$, the willow bark extract was analyzed in terms of content in polyphenols, flavones and salicylic derivatives, as well as antioxidant activity. The concentration of total salicylic derivatives was determined by high pressure liquid chromatography (HPLC), while the antioxidant potential and free radical scavenging activity of $S$. alba extract was assessed using the CUPRAC method.

For studying the effect of certain natural aspirin-like compounds with antiglycosylating activity on the in vitro non-enzymatic glycation of human hemoglobin, the human erythrocytes isolated from whole peripheral blood according to the protocol described by Arias et al. [4] were incubated with different concentrations of fructose/glucose (10,50, $100 \mathrm{mM})$ and S. alba extract for 5, 7, 10 and 14 days.

The esterase activity of hemoglobin was determined by the method described by Sen et al. using p-nitrophenyl acetate as substrate [5], while the determination of the peroxidase activity of human hemoglobin was performed according to the method used by Trinder [6], by measuring the increase in absorbance at $510 \mathrm{~nm}$, following the decomposition of $\mathrm{H}_{2} \mathrm{O}_{2}$, using 4-aminoantipyrine as a hydrogen donor. 
The 1st International Electronic Conference on Antioxidants in Health and Disease, 1-15 December 2020

To determine the absorption in THz of human hemoglobin solutions, the protocol described by Mernea et al. [7] was used and two sets of measurements were performed: without sample, when the reference spectrum was recorded (Eref $(\omega)$ ) and with sample, when the spectrum of the sample was recorded (Esam $(\omega)$ ); the absorption of the samples was determined by the ratio of the two measurements.

\section{Results}

In terms of chemical composition, white willow bark contains tannins (3-10\%), salicin (0.3$0.8 \%$ ), salicylic acid, saligenol, waxes, resins and other flavonic derivatives and a series of oxalates with important pharmacodynamic effects on the human body. HPLC analysis of the willow bark extract obtained in our study resulted in a content of $0.011 \mathrm{mg} / \mathrm{mL}$ salicylic derivatives, expressed as salicin.

The potential antiglycosylating effect of willow bark extract is due to significant concentrations of polyphenols and flavonoid compounds which also provide a high antioxidant activity (Table 1), salicylates also having the role of maintaining oxidative stress at a low level that does not disrupt the physiological processes at the cellular level.

Table 1. Physicochemical determinations of antiglycosylating compounds and antioxidant activity from willow bark extract.

\begin{tabular}{cc}
\hline Method & Amount Detected \\
\hline Polyphenols (gallic acid) & $2.34 \pm 0.62 \mathrm{mg} / \mathrm{mL}$ \\
Flavonoids (rutin) & $0.14 \pm 0.03 \mathrm{mg} / \mathrm{mL}$ \\
Antioxidant activity (Trolox) & $16.92 \pm 2.16 \mathrm{mmol} / \mathrm{mL}$ \\
\hline
\end{tabular}

\section{Discussion}

Salicin has always been considered the main active substance in willow bark and therefore used as a biological marker of its activity, even if Salicis cortex extracts contain up to $20 \%$ condensed polyphenols and tannins. In the case of aqueous extracts, the predominant flavonoids are the glycosides of naringenin, isosalipurpuroside or eriodictiol, respectively, and the major polyphenolic fraction consists of flavan-3-oils (catechin, epicatechin, galocatechin, etc.) and a series of procyanidins $[8]$.

\section{Conclusions}

Our study showed that willow bark extract has positively influenced the changes suffered by the peroxidase activity of hemoglobin, maintaining the initial value in the case of the glycosylation process and significantly attenuating its decrease after fructosylation. Moreover, if S. alba extract had a beneficial effect on the changes in peroxidase activity generated by both glucose and fructose binding, in the case of esterase activity it was observed that they had a significant antiglycosylating potential only in the case of fructose. Therefore, the salicylic compounds present in the bark extract of $S$. alba can be successfully used as natural substituents of aspirin to prevent glycosylation processes and AGE formation.

Acknowledgments: This research was funded by the Executive Agency for Higher Education, Research, Development and Innovation Funding (UEFISCDI), within project no. PN-11l-P2-2.1-PED-2019-1471.

Conflicts of Interest: The authors declare no conflict of interest. The funders had no role in the design of the study; in the collection, analyses, or interpretation of data; in the writing of the manuscript, or in the decision to publish the results. 
The 1st International Electronic Conference on Antioxidants in Health and Disease, 1-15 December 2020

\section{References}

1. Bakhti, M.; Habibi-Rezaei, M.; Moosavi-Movahedi, A.A.; Khazaei, M.R. Consequential alterations in haemoglobin structure upon glycation with fructose: Prevention by acetylsalicylic acid. J. Biochem. 2007, 141, 827-833, doi:10.1093/jb/mvm096.

2. Odjakova, M.; Popova, E.; Al Sharif, M.; Mironova, R. Plant-derived agents with anti-glycation activity. In Glycosylation; Petrescu, S., Eds.; InTechOpen: London, UK, 2012; Volume 1, pp. 223-256.

3. Baltazar, M.T.; Dinis-Oliveira, R.J.; Duarte, J.A.; Bastos, M.L.; Carvalho, F. Antioxidant properties and associated mechanisms of salicylates. Curr. Med. Chem. 2011, 18, 3252-3264, doi:10.2174/092986711796391552.

4. Arias, M.; Quijano, J.C.; Haridas, V.; Gutterman, J.U.; Lemeshko, V.V. Red blood cell permeabilization by hypotonic treatments, saponin, and anticancer avicins. Biochim. Biophys. Acta 2010, 1798, 1189-1196, doi:10.1016/j.bbamem.2010.03.018.

5. Sen, S.; Bose, T.; Roy, A.; Chakraborti, A.S. Effect of non-enzymatic glycation on esterase activities of hemoglobin and myoglobin. Mol. Cell Biochem. 2007, 301, 251-257, doi:10.1007/s11010-007-9418-5.

6. Trinder, P. Determination of glucose in blood using glucose oxidase with an alternative oxygen acceptor. Ann. Clin. Biochem. 1969, 6, 24-31, doi:10.1177/000456326900600108.

7. Mernea, M.; Ionescu, A.; Vasile, I.; Nica, C.; Stoian, G.; Dascalu, T.; Mihailescu, D.F. In vitro human serum albumin glycation monitored by Terahertz spectroscopy. Opt Quant Electron 2015, 47, 961-973, doi:10.1007/s11082-015-0129-y.

8. Nahrstedt, A.; Schmidt, M.; Jäggi, R.; Jürgen, M.; Khayyal, M.T. Willow bark extract: The contribution of polyphenols to the overall effect. Wien Med Wochenschr 2007, 157, 348-351, doi:10.1007/s10354-007-0437-3.

Publisher's Note: MDPI stays neutral with regard to jurisdictional claims in published maps and institutional affiliations.

(C) 2020 by the authors; licensee MDPI, Basel, Switzerland. This article is an open access article distributed under the terms and conditions of the Creative Commons by Attribution (CC-BY) license (http://creativecommons.org/licenses/by/4.0/). 